\title{
BOUNDARY BEHAVIOR OF GENERALIZED POISSON INTEGRALS FOR THE HALF-SPACE AND THE DIRICHLET PROBLEM FOR THE SCHRÖDINGER OPERATOR
}

\author{
ALEXANDER I. KHEIFITS
}

(Communicated by J. Marshall Ash)

\begin{abstract}
The boundary properties are investigated for the generalized Poisson integral

$$
u(X)=\int_{\mathbb{R}^{n}} k(X, y) f(y) d y,
$$

where $X$ is a point of the upper half-space $\mathbb{R}_{+}^{n+1}, f \in L^{\mathbf{p}}\left(\mathbb{R}^{n}\right), 1 \leq \mathbf{p} \leq \infty$, and the kernel $k$ has some special properties. Our results imply the known boundary properties of the harmonic Poisson integrals on the half-space. As an application we derive a solution of the Dirichlet problem for the operator $-\Delta+c(X), X \in \mathbb{R}_{+}^{n+1}$, with boundary data $f \in L^{\mathbf{P}}\left(\mathbb{R}^{n}\right)$.
\end{abstract}

\section{INTRODUCTION AND STATEMENT OF RESULTS}

In their well-known paper [1] Fefferman and Stein extended the classical theory of Hardy spaces $\mathbf{H}^{\mathbf{p}}$ on harmonic functions in the half-space

$$
\mathbb{R}_{+}^{n+1}=\left\{X=\left(x, x_{n+1}\right): x \in \mathbb{R}^{n}, x_{n+1}>0\right\}, \quad n \geq 1 .
$$

In this question the Poisson integral plays an essential role. The Laplace operator $\Delta$ has constant coefficients; hence the Poisson integral, i.e., a normal derivative of the Green function for the half-space, depends on the difference of arguments and consequently in the harmonic case the Poisson integral is a convolution. In the same article [1] the authors considered the boundary behavior of more general convolution integrals.

In the next step the problem appears to investigate the boundary behavior of nonconvolution integrals of the kind

$$
u(X)=\int_{\mathbb{R}^{n}} k(X, y) f(y) d y, \quad X \in \mathbb{R}_{+}^{n+1},
$$

which in this context it is natural to call the generalized Poisson integrals.

If $k$ in (1) is the Poisson kernel for the Laplacian, then the following result is known for the function (1) (see [2, Chapters 3 and 7]).

Received by the editors July 29, 1991 and, in revised form, January 10, 1992.

1991 Mathematics Subject Classification. Primary 31C99, 35J10, 35 J25. 
Theorem A. Let $f \in L^{\mathbf{p}}\left(\mathbb{R}^{n}\right), 1 \leq \mathbf{p} \leq \infty$, and let $B(x, r)$ be a ball in $\mathbb{R}^{n}$ with center $x$ and radius $r, \omega_{n}$ the volume of $B(0,1)$, and $(M f)(x)=$ $\sup _{r>0}\left(\omega_{n} r^{n}\right)^{-1} \int_{B(x, r)}|f(y)| d y$ the maximal function. Then

$1^{\circ}$. $\sup _{0<x_{n+1}<\infty}|u(X)| \leq(M f)(x) \forall x \in \mathbb{R}^{n}$, as before, here $X=\left(x, x_{n+1}\right)$;

$2^{\circ} . \lim _{x_{n+1} \rightarrow 0} u(X)=f(x)$ for Lebesgue almost every $x \in \mathbb{R}^{n}$;

$3^{\circ}$. for $\mathbf{p}<\infty, \lim _{x_{n+1} \rightarrow 0}\|u(X)-f(x)\|=0$.

Here and in the sequel $\|\cdot\|$ denotes the norm in $L^{\mathbf{p}}\left(\mathbb{R}^{n}\right)$.

Actually in [2] the generalization of Theorem A, particularly when the Poisson kernel is replaced by any approximate identity, was proved. In addition the statement was proved in [2] that in $1^{\circ}$ and $2^{\circ}$ the condition $x_{n+1} \rightarrow 0$ (i.e., $X \rightarrow X$ along the normal to the boundary $\mathbb{R}^{n}$ of $\mathbb{R}_{+}^{n+1}$ ) may be interchanged to the tending $X \rightarrow x$ as $X$ belongs to the cone

$$
\Gamma_{\alpha}\left(x_{0}\right)=\left\{X \in \mathbb{R}_{+}^{n+1}:\left|x-x_{0}\right|<\alpha x_{n+1}\right\}, \quad \alpha>0, x_{0} \in \mathbb{R}^{n} .
$$

Our aim is to extend Theorem A on the integrals (1) where the kernel $k(X, y)$ is defined and measurable on the Cartesian product $\mathbb{R}_{+}^{n+1} \times \mathbb{R}^{n}$. We investigate the boundary behavior of (1) and apply our results to the Dirichlet problem for the Schrödinger operator $-\Delta+c(X) I$ in the half-space, $I$ being the identity operator. Now we state our results. All proofs will be given in $\S 2$.

Proposition 1. Fix a point $x \in \mathbb{R}^{n}$. Suppose the kernel $k(X, y)$ has the summable majorant $\psi\left(x_{n+1}, \cdot\right) \in L\left(\mathbb{R}^{n}\right)$, depending only on $|x-y|:|k(X, y)| \leq$ $\psi\left(x_{n+1},|x-y|\right)$ for all $X=\left(x, x_{n+1}\right), 0<x_{n+1}<h$, with some $h>0$. Let $\psi\left(x_{n+1}, r\right)$ decrease monotonically for $0<r<\infty$ and

$$
A\left(x_{n+1}\right) \equiv \int_{\mathbb{R}^{n}} \psi\left(x_{n+1},|y|\right) d y=O(1) \text { as } x_{n+1} \rightarrow 0 .
$$

If $f \in L^{\mathbf{p}}\left(\mathbb{R}^{n}\right), 1 \leq \mathbf{p} \leq \infty$, then the following is valid for the function (1):

$$
\limsup _{x_{n+1} \rightarrow 0}|u(X)| \leq A(M f)(x),
$$

where $A=\lim \sup _{x_{n+1} \rightarrow 0} A\left(x_{n+1}\right)$.

Corollary. If the conditions of the proposition are satisfied with the same $\psi$ at almost every point $x \in \mathbb{R}^{n}$ and $1<\mathbf{p} \leq \infty$, then

$$
\limsup _{x_{n+1} \rightarrow 0}\left\|u\left(\cdot, x_{n+1}\right)\right\| \leq A\|f\|, \quad A=\text { const . }
$$

If $\sup _{x_{n+1}>0} A\left(x_{n+1}\right)<\infty$ and $\mathbf{p}>1$, then

$$
\sup _{x_{n+1}>0}\left\|u\left(\cdot, x_{n+1}\right)\right\| \leq A_{\mathbf{p}}\|f\| \text {. }
$$

Proposition 2. Let the conditions of the previous proposition be satisfied for Lebesgue almost every $x \in \mathbb{R}^{n}$. Besides let there exist the limit

$$
\lim _{x_{n+1} \rightarrow 0} \int_{\mathbb{R}^{n}} k(X, y) d y=1
$$

for Lebesgue a.e. $x \in \mathbb{R}^{n}$ and the limit

$$
\lim _{x_{n+1} \rightarrow 0} \int_{|x-y| \geq \delta}|k(X, y)| d y=0
$$


for every $\delta>0$. Then there exists the limit

$$
\lim _{x_{n+1} \rightarrow 0} u(X)=f(x) \text { a.e. on } \mathbb{R}^{n} \text {. }
$$

Remark. If in Propositions 1 and 2 the condition $x_{n+1} \rightarrow 0$ is replaced by $\Gamma_{\alpha}\left(x_{0}\right) \ni X \rightarrow x_{0}$, then all the assertions will remain valid as $\Gamma_{\alpha}\left(x_{0}\right) \ni X \rightarrow x_{0}$.

Proposition 3. Suppose all conditions of Proposition 2 are satisfied and, in addition, the condition (2) is strengthened to

$$
\lim _{x_{n+1} \rightarrow 0} \int_{|y| \geq \delta} \psi\left(x_{n+1},|y|\right) d y=0 \quad \forall \delta>0 .
$$

If $1 \leq \mathbf{p}<\infty$ and $\left|\int_{\mathbb{R}^{n}} k(X, y) d y-1\right| \leq$ const $<\infty$ uniformly in $X \in$ $\mathbb{R}^{n} \times(0, h)$, then there exists the limit

$$
\lim _{x_{n+1} \rightarrow 0}\|u(X)-f(x)\|=0 .
$$

Evidently all our conjectures are fulfilled for the classical Poisson kernel

$$
\gamma_{n+1} x_{n+1}\left\{|x-y|^{2}+x_{n+1}^{2}\right\}^{-(n+1) / 2}, \quad \gamma_{n+1}=\text { const } ;
$$

hence Propositions 1-3 imply Theorem A. Our assumptions are valid too for the kernels

$$
\left(x_{n+1}^{2} /\left(|x-y|^{2}+x_{n+1}^{2}\right)\right)^{\lambda n / 2} \cdot x_{n+1}^{-n}
$$

and

$$
\left(x_{n+1} /\left(|x-y|+x_{n+1}\right)\right)^{\lambda n} \cdot x_{n+1}^{-n}
$$

with $\lambda>1$, and Proposition 1 implies the case $z=0$ and real $\lambda>1$ of Lemma 3.3 by Johnson [3].

Now let us consider the operator $L_{c}=-\Delta+c(X) I$, where the function (potential) $c(X) \geq 0$ in $\mathbb{R}_{+}^{n+1}$ and such that $c \in L^{s}$ in some neighbourhood of each finite point $X \in \mathbb{R}_{+}^{n+1} \cup \mathbb{R}^{n}$ with certain $s>(n+1) / 2$ for $n \geq 3$ and $s=2$ for $n=1,2$. Besides we suppose that $c(X)$ has summable majorant depending only on $|X-y|$ in some vicinity of any boundary point $y \in \mathbb{R}^{n}$. Under these assumptions it is known that the operator $L_{c}$ on $L_{2}\left(\mathbb{R}_{+}^{n+1}\right)$ has a Green function $G(X, Y)$ in $\mathbb{R}_{+}^{n+1}$ with analytic properties necessary in the sequel. Consequently Propositions $1-3$ and the results of $[4,5]$ imply the following.

Theorem. Suppose $f \in L^{\mathbf{p}}\left(\mathbb{R}^{n}\right), 1 \leq \mathbf{p} \leq \infty$. Then the Dirichlet problem

$$
\begin{cases}-\Delta u+c(X) u, & X \in \mathbb{R}_{+}^{n+1}, \\ u(x)=f(x) & \text { a.e. on } \mathbb{R}^{n}\end{cases}
$$

has the solution

$$
u(X)=\int_{\mathbb{R}^{n}} \frac{\partial G(X, y)}{\partial n(y)} f(y) d y, \quad X \in \mathbb{R}_{+}^{n+1},
$$

which satisfies all the conclusions of Propositions 1-3. Here $\partial / \partial n$ is a derivative along an inner normal to $\mathbb{R}^{n}$. Moreover, this solution $u$ is Hölder continuous with exponent $2-n / s$ if $n / 2<s \leq n$ and its gradient $\nabla u$ is Hölder continuous with exponent $1-n / s$ if $s>n$ in all points $X \in \mathbb{R}_{+}^{n+1}$. For potentials under consideration this assertion makes more precise Simon's results about Hölder 
continuity of the solutions to the Schrödinger equation $L_{c} u=0[6$, Theorems C.2.4 and C.2.5, p. 497].

Of course this solution of problem (3) is not unique. The uniqueness is valid only with a priori growth estimates of the solution at infinity; this question will be considered elsewhere.

\section{Demonstrations}

We essentially use some ideas of Stein's book [2].

Proof of Proposition 1. By assumption

$$
I \equiv \int_{\mathbb{R}^{n}} \psi\left(x_{n+1},|y-x|\right) d y<\infty .
$$

If we introduce spherical coordinates in $\mathbb{R}^{n}$ with pole $x$, then $I=$ $n \omega_{n} \int_{0}^{\infty} r^{n-1} \psi\left(x_{n+1}, r\right) d r$. The monotonicity of $\psi$ implies $r^{n} \psi\left(x_{n+1}, r\right) \rightarrow$ 0 as $r \rightarrow 0$ and $r \rightarrow \infty$. Now integration by parts leads to the equality $I=\omega_{n} \int_{0}^{\omega} r^{n} d\left\{-\psi\left(x_{n+1}, r\right)\right\}$. Denote (see [2])

$$
\lambda(r)=\int_{|t-x|=r} f(t) d \sigma(t)
$$

and

$$
\Lambda(r)=\int_{|x-y| \leq r}|f(y)| d y=\int_{0}^{r} t^{n-1} \lambda(t) d t .
$$

Then $\Lambda(r) \leq \omega_{n} r^{n}(M f)(x)$. Hence if $(M f)(x)<\infty$, then for $0<x_{n+1}<h$,

$$
\lim \Lambda(r) \psi\left(x_{n+1}, r\right)=0, \quad r \rightarrow 0, r \rightarrow \infty .
$$

Now we can estimate the function $u$ :

$$
|u(X)| \leq \int_{\mathbb{R}^{n}}|k(X, y)||f(y)| d y \leq \int_{0}^{\infty} \Lambda(r) d_{r}\left\{-\psi\left(x_{n+1}, r\right)\right\}
$$

with regard to (4). From this we have

$$
|u(X)| \leq \omega_{n}(M f)(x) \int_{0}^{\infty} r^{n} d_{r}\left\{-\psi\left(x_{n+1}, r\right)\right\},
$$

and after back integration by parts the inequality $|u(X)| \leq A\left(x_{n+1}\right)(M f)(x)$ follows. To finish the proof it remains to let $x_{n+1} \rightarrow 0$. Q.E.D.

Proof of Proposition 2. Transform the difference

$$
\begin{aligned}
u(X)-f(x)= & \int_{|y-x|<\delta} k(X, y)\{f(y)-f(x)\} d y \\
& +\int_{|y-x| \geq \delta} k(X, y)\{f(y)-f(x)\} d y+f(x)\left\{\int_{\mathbb{R}^{n}} k(X, y) d y-1\right\} \\
& \equiv I_{1}+I_{2}+I_{3}
\end{aligned}
$$

and estimate every term separately.

Let $x$ be a point of the Lebesgue set of the function $f$; then $|f(x)|<\infty$. If $x$ does not belong to other exceptional sets of zero measure (see the statement of the proposition), i.e., $x$ belongs to the set of full measure in $\mathbb{R}^{n}$, then $\lim _{x_{n+1} \rightarrow 0} I_{3}=0$ by virtue of (2). Similarly, $\lim _{x_{n+1} \rightarrow 0} f(x) \int_{|y-x| \geq \delta} k(X, y) d y$ 
$=0$. We estimate the integral $I_{21}=\int_{|y-x| \geq \delta} k(X, y) f(y) d y$ by the Hölder inequality:

$$
\left|I_{21}\right| \leq\|f\|\left\{\int_{|y-x| \geq \delta}|k(X, y)|^{\mathbf{q}} d y\right\}^{1 / \mathbf{q}}, \quad \mathbf{q}=\mathbf{p} /(\mathbf{p}-1) \geq 1 .
$$

The inequality $|k(X, y)|<1$ is valid asymptotically by virtue of the monotonicity and integrability of the majorant $\psi$. Hence $|k(X, y)|^{\mathbf{q}} \leq|k(X, y)|$ asymptotically and

$$
\left|I_{21}\right| \leq \text { const }\|f\|\left\{\int_{|y-x| \geq \delta}|k(X, y)| d y\right\}^{1 / \mathbf{q}} \rightarrow 0 \quad \text { as } x_{n+1} \rightarrow 0 .
$$

To estimate $I_{1}$ we introduce the function as in [2]

$$
g(y)= \begin{cases}f(y)-f(x) & \text { if }|y-x|<\delta, \\ 0 & \text { if }|y-x| \geq \delta .\end{cases}
$$

For every $\varepsilon>0$ we can choose $\delta=\delta(\varepsilon)$ such that $(M g)(x)<\varepsilon$ because $x$ is the point of the Lebesgue set of $f$. Therefore Proposition 1 implies $\left|I_{1}\right| \leq A(M g)(x)<A \varepsilon$, where $\varepsilon$ may be arbitrarily small. Q.E.D.

Proof of Proposition 3. We have $\|u(X)-f(x)\| \leq\left\|I_{1}\right\|+\left\|I_{2}\right\|+\left\|I_{3}\right\|$. First

$$
\begin{aligned}
\left|I_{1}\right| & =\left|\int_{|y|<\delta} k(X, y)\{f(y+x)-f(x)\} d y\right| \\
& \leq \int_{|y| \leq \delta} \psi\left(x_{n+1},|y|\right)|f(y+x)-f(x)| d y,
\end{aligned}
$$

but $\psi\left(x_{n+1}, r\right)$ does not depend on $x$; hence

$$
\left\|I_{1}\right\| \leq \int_{|y|<\delta} \psi\left(x_{n+1}, r\right)\|f(y+\cdot)-f(\cdot)\| d y .
$$

It is known that the relation $\Delta(y) \equiv\|f(y+\cdot)-f(\cdot)\|=o(1)$ as $|y|<\delta, \delta \rightarrow 0$. Hence for arbitrary fixed $\varepsilon>0$ one can choose $\delta>0$ such that $\Delta(y)<\varepsilon$ and consequently

$$
\left\|I_{1}\right\| \leq \varepsilon \int_{|y|<\delta} \psi\left(x_{n+1},|y|\right) d y \leq A\left(x_{n+1}\right) \varepsilon \leq A \varepsilon .
$$

Now we fix $\delta>0$ and estimate

$$
\begin{aligned}
\left\|I_{2}\right\| & \leq\left\|\int_{|y-x| \geq \delta} \psi\left(x_{n+1},|y-x|\right)|f(y)-f(x)| d y\right\| \\
& \leq \int_{|y| \geq \delta} \psi\left(x_{n+1},|y|\right)\|f(y+\cdot)-f(\cdot)\| d y \\
& \leq 2\|f\| \int_{|y| \geq \delta} \psi\left(x_{n+1},|y|\right) d y=o(1), \quad x_{n+1} \rightarrow 0 .
\end{aligned}
$$

To estimate $\left\|I_{3}\right\|$ (now $\mathbf{p}<\infty$ ) we use the representation (see [2]) $f=$ $f_{1}+f_{2}$, where $f_{1}$ is a continuous function with compact support and $\left\|f_{2}\right\|<\varepsilon$. Hence $\sup \left|f_{1}\right|<\infty$ and the function with compact support

$$
\left\{\left|f_{1}(x)\right|\left|\int_{\mathbb{R}^{n}} k(X, y) d y-1\right|\right\}^{\mathbf{p}}
$$


has a summable majorant. Now from the Lebesgue theorem about dominated convergence we have

$$
\lim _{x_{n+1} \rightarrow 0} \int_{\mathbb{R}^{n}}\left|f_{1}(x)\right|^{\mathbf{p}}\left|\int_{\mathbb{R}^{n}} k(X, y) d y-1\right|^{\mathbf{p}} d x=0 .
$$

Finally,

$$
\left\|f_{2}(x)\left(\int_{\mathbb{R}^{n}} k(X, y) d y-1\right)\right\| \leq \text { const }\left\|f_{2}\right\| \leq \text { const } \varepsilon . \quad \text { Q.E.D. }
$$

\section{ACKNOWLEDGMENT}

The author is grateful to the referee for his essential remarks concerning this manuscript.

\section{REFERENCES}

1. C. Fefferman and E. M. Stein, $\mathbf{H}^{\mathbf{p}}$ spaces of several variables, Acta Math. 129 (1972), 137-193.

2. E. M. Stein, Singular integrals and differential properties of functions, Princeton Univ. Press, Princeton, NJ, 1970.

3. R. Johnson, Application of Carleson measures to partial differential equations and Fourier multiplier problems, Harmonic Analysis, Lecture Notes in Math., vol. 992, Springer-Verlag, Berlin, Heidelberg, New York, and Tokyo, 1983, pp. 16-72.

4. A. I. Kheifits, Subfunctions of the Schrödinger operator. 2, Rostov State Univ., Rostov-onDon, 1989; RZhMat 1989: 45541Dep.

5. _ Subfunctions of the Schrödinger operator. 3, Capacity and its Applications, Rostov State Univ., Rostov-on-Don, 1990; RZhMat 1990: 116383Dep.

6. B. Simon, Schrödinger semigroups, Bull. Amer. Math. Soc. (N.S.) 7 (1982), 447-526.

Rostov State University, ul. Sodruzhestva 37, kv. 198, Rostov-on-Don, Republic of RUSSIA 